\title{
La simulation avec des moyens limités: des possibilités pédagogiques sans limites
}

\author{
Simulation with limited resources: unlimited educational possibilities
}

Alors que la simulation est devenue une méthode d'enseignement et d'apprentissage recommandée pour la formation des professionnels de la santé, aussi bien en formation initiale qu'en formation continue, son financement reste un des écueils majeurs à son développement dans notre milieu, notamment lorsque l'on recours à des dispositifs de haute technologie. Il pourrait dès lors paraître paradoxal de parler de simulation dans le contexte économique des pays émergents. Le présent numéro de la revue propose deux contributions consacrées à des expériences de développement de la simulation, mises en œuvre dans deux facultés de médecine d'Algérie. Elles sont illustratives d'une approche pragmatique et raisonnée de ce que la simulation peut apporter, même sans moyens considérables.

La première contribution rapporte une étude d'évaluation d'un dispositif de simulation mis en place par la Faculté de médecine d'Annaba et le service d'anesthésieréanimation du Centre hospitalier universitaire d'Annaba, au sein d'un cursus de formation spécialisée en anesthésie-réanimation. Dans ce contexte, les auteurs ont souhaité documenter l'impact pédagogique d'une formation par simulation proposée à un groupe de 30 internes pour l'apprentissage des procédures de gestion des voies aériennes supérieures [1]. L'objectif était de comparer les performances de deux groupes d'internes, l'un ayant bénéficié d'une formation utilisant la simulation, l'autre bénéficiant uniquement de l'enseignement par compagnonnage traditionnel lors de procédures d'anesthésie sur de vrais patients. Après une session d'enseignement théorique commune, les internes étaient répartis par tirage au sort dans un groupe «simulation» et un groupe «sans simulation». La formation par simulation était délivrée en deux parties successives :

- un entraînement pratique en vue de l'acquisition d'habiletés techniques de base sur des mannequins de basse technologie;
- la mise en situation contextualisée sur un mannequin de haute technologie préalablement paramétré pour simuler, de façon non programmée, plusieurs situations emblématiques de la gestion des voies aériennes supérieures au cours de la pratique d'anesthésieréanimation.

Chaque session se déroulait selon le modèle «briefingséance de simulation-débriefing ». Les performances des deux groupes d'internes étaient évaluées de façon répétée par les superviseurs lors de situations de pratique réelle d'anesthésie, à l'aide de grilles préétablies. Les scores obtenus par le groupe «enseignement par simulation»se sont avérés supérieurs à ceux du groupe «enseignement classique », pour la majorité des points clés jugés comme indispensables pour une gestion adéquate des voies aériennes supérieures. Les auteurs en déduisent que l'utilisation de la simulation a permis à leurs internes de développer des capacités mobilisables dans des tâches authentiques à un niveau supérieur de performances, comparativement aux internes formés selon une approche expérientielle traditionnelle.

Bien que ces résultats ne portent que sur un effectif restreint, cette étude vient confirmer les données probantes déjà disponibles concernant deux points essentiels, à savoir :

- l'entraînement sur simulateur de basse technologie est aussi efficace que celui recourant à des simulateurs de haute technologie pour l'acquisition d'habiletés techniques de base [2];

- l'acquisition d'un geste ou d'une procédure technique est un processus complexe dont la composante essentielle est cognitivo-motrice.

Tout programme de formation à une procédure technique, surtout si elle est complexe, devrait donc proposer un entraînement par fractionnement de la procédure, largement supérieur en termes de transférabilité des habiletés que les entraînements par procédures 
complètes [3]. En ce sens, la justesse de l'approche pédagogique proposée par les auteurs pour l'apprentissage de la gestion des voies aériennes supérieures est à souligner.

Cette procédure est par ailleurs une des plus emblématiques de la pratique d'anesthésie-réanimation et de la médecine d'urgence. L'utilisation de la simulation pour l'entraînement aux habiletés techniques requises, pour l'entraînement individuel et en équipe à la gestion des risques et des complications, est devenue une pratique standardisée dans les programmes de formation d'anesthésie-réanimation dans les pays développés. Le choix des auteurs de privilégier un dispositif de simulation dans leur programme pédagogique est dans ce cadre parfaitement justifié. Leur réflexion pour proposer l'outil de simulation le plus adapté à leur contexte économique est également à souligner et doit être encouragée.

La seconde contribution est produite par les enseignants de la Faculté de médecine de Mostaganem et concerne un programme de formation par la simulation en formation initiale au niveau pré-gradué [4]. Les auteurs partent du constat que le contexte parfois imprévisible du stage hospitalier et d'externat n'offre pas toujours toutes les garanties - en termes de sécurité pour le patient et en termes de sérénité pour l'étudiant - pour un apprentissage optimal de la sémiologie et de l'examen clinique. Dans ce cadre, les auteurs ont mené un travail comparatif concernant les performances réalisées dans le cadre d'une évaluation formelle, impliquant respectivement des étudiants soumis à un programme de simulation associé au stage hospitalier et des étudiants ne bénéficiant que d'une immersion hospitalière. Ce travail a été mené au sein d'une promotion entière d'étudiants de troisième année, répartie de manière randomisée en deux groupes qui ont suivi, respectivement, un programme de gestes procéduraux avec simulation et le programme des examens physiques sans simulation. Une évaluation des apprentissages a été réalisée par examen clinique objectif structuré (ECOS) pour les acquisitions pratiques et par un test de connaissances avec questions à choix multiples pour les acquisitions théoriques. Il faut noter que les sessions de simulation s'appuyaient là aussi, principalement, sur des dispositifs de basse technologie pour les habiletés techniques de base et sur des jeux de rôles pour l'examen clinique neurologique et ostéo-articulaire. Seule l'auscultation cardio-pulmonaire était enseignée sur simulateur de haute technologie. Globalement, les étudiants bénéficiant d'apprentissage par simulation associée à l'immersion hospitalière se sont avérés plus performants que les étudiants du groupe témoin, aussi bien pour les acquisitions pratiques que pour les connaissances théoriques.

Cette étude a le mérite d'aborder une problématique largement partagée au sein de nos facultés et qui concerne un très grand nombre de programmes de formation initiale, quel que soit l'environnement économique. Deux aspects peuvent être mis en exergue:

- l'hétérogénéité des expositions cliniques des étudiants au sein des stages hospitaliers, ainsi que l'hyperspécialisation des services dans lesquels s'effectuent les stages rendent compte de la difficulté de l'immersion hospitalière proposée en début de cursus à répondre adéquatement à son objectif pédagogique officiel, à savoir l'apprentissage de la sémiologie générale et des techniques d'examen physique;

- sous réserve d'une confirmation par des études à plus long terme, l'apprentissage contextualisé que permet la simulation est un facteur de meilleure rétention des connaissances théoriques.

Ainsi, la simulation en santé ne doit pas être considérée exclusivement comme un outil conçu pour le développement professionnel continu et la gestion des risques. Elle favorise l'apprentissage dit «actif», même pour des habiletés de base, qu'il s'agisse du «savoir», du «savoir-être » et du «savoir-faire ». Elle se place en tant que solution potentielle pour réduire l'écart existant entre le niveau de compétence clinique requis et le faible niveau d'exposition. Son intégration dans les programmes de formation initiale, y compris en tout début de cursus, est ainsi justifiée, de façon complémentaire aux autres activités d'apprentissage, tout en prenant soin d'utiliser des dispositifs et appareils de technicité et de sophistication adaptés aux objectifs pédagogiques [5]. Le travail de l'équipe de Mostaganem en est une parfaite illustration.

Ces deux contributions viennent enrichir la réflexion plus globale sur la place de la simulation en santé au sein des programmes de formation, qui pourrait s'articuler autour de deux axes:

- le premier concerne la façon d'envisager la simulation comme outil pédagogique et son intégration dans un cursus. En effet, il est toujours utile de rappeler que l'implantation d'un programme de formation par la simulation doit nécessairement être précédée par une étape de réflexion sur les besoins pédagogiques et sur l'adaptation des outils en fonction des objectifs, du contexte et des apprenants. L'aspect «high tech» et novateur que peuvent parfois prendre les outils de simulation, sans évoquer la pression parfois exercée par l'industrie, ne doit pas faire perdre de vue qu'il s'agit avant tout d'outils au service de la formation. Qu'il s'agisse de formation initiale ou de développement professionnel, la simulation doit être intégrée dans un environnement d'apprentissage en complément des outils existants et non simplement ajoutée. De plus, l'articulation de la simulation avec les différentes modalités d'apprentissage doit être systématiquement évaluée et valorisée, dans le sens d'une synergie pédagogique ;

- le second axe de réflexion, dans le prolongement du premier, concerne la mise en œuvre de la simulation avec des moyens limités dans les pays «émergents ». Le coût financier de la simulation est un élément essentiel à prendre en compte lors de son implantation.

Les deux articles sont exemplaires d'une nécessaire documentation «écologique », à intégrer dans la réflexion pédagogique quand il s'agit de simulation. Ils démontrent que l'implantation de la simulation dans les curriculums peut être viable dans les pays à économie émergente. 
De telles publications pourraient d'ailleurs aider les responsables institutionnels des facultés à défendre les demandes d'investissement auprès de leurs gestionnaires.

Ils démontrent également parfaitement que même si les simulateurs de basse technologie «faits maison» ne permettent pas de tout apprendre, ils peuvent être des outils efficaces d'apprentissage, voire d'évaluation, et que la création de nouveaux modèles doit être encouragée, dans les situations ou les structures ou les moyens financiers ne sont pas extensibles [2]. Les exemples sont multiples où des moyens extrêmement simples ont pu être utilisés pour apprendre des gestes comme les sutures ou les ponctions sur différents fruits - tomates, oranges, bananes -, ou parties d'animaux - pattes de poulet ou jarret de porc-, pour n'en citer que quelques-uns [6]. Par ailleurs, la création de tels outils stimule sans aucun doute l'imagination et la cohésion des équipes formatrices. Les jeux de rôle, la participation de patients simulés peuvent également contribuer de façon peu onéreuse au développement de programmes de simulation dans le domaine de la sémiologie, de l'apprentissage des habiletés relationnelles ou de l'entraînement en équipe.

Dans les situations concernant des structures dont les moyens financiers ne sont pas extensibles, le développement de l'accès à Internet a également son rôle à jouer. La diffusion peu onéreuse de programmes de formation par simulation à distance, qu'ils soient dédiés à un geste technique ou qu'il s'agisse de jeux sérieux - serious games-, représente actuellement un excellent moyen de formation par simulation.

Finalement, la simulation est une source d'échanges et de collaborations multidisciplinaires et interprofessionnelles. Cette mission inclut la mise en œuvre de projets de co-développement entre centres de simulations de différents milieux. Les sociétés savantes issues du monde francophone, impliquées dans l'éducation médicale, donc en simulation, telles que la Société internationale francophone d'éducation médicale (SIFEM) et la Société francophone de simulation en santé (SoFraSimS; www. sofrasims.org) sont les structures les plus à même de remplir ces missions.

Louis SIBERT ${ }^{1,2, *}$

${ }^{1}$ Service d'urologie, Centre hospitalier universitaire de Rouen, 1, rue de Germont, 76000 Rouen, France

${ }^{2}$ Medical Training Center Rouen-Normandie, 20, rue Marie-Curie, 76000 Rouen, France

*Mailto: Louis.Sibert@chu-rouen.fr

\section{Références}

1. Bouchareb M, Bouaziz M, Djebien M, Saidani M. Formation de résidents en anesthésie-réanimation à la gestion des voies aériennes. Évaluation d'un dispositif pédagogique recourant à la simulation dans une faculté de médecine en Algérie. Pédagogie Médicale 2020. https://doi.org/10.1051/pmed/ 2020030.

2. Bazin JÉ, Péan D. Simulation avec des moyens limités: simulation en pays émergents. In: Boet S, Savoldelli G, Granry JC (eds.). La simulation en santé de la théorie à la pratique. Paris: Springer, 2013.

3. Sakai K, Kitaguchi K, Hikosaka O. Chunking during human visuomotor sequence learning. Exp Brain Res 2003;152: 229-42.

4. Mohammed MR, Maiza A, Seddiki MS, Mokhtari L. Les effets de l'intégration de la simulation sur l'apprentissage des gestes procéduraux de base et de l'examen physique en stage hospitalier dans le cursus pré-gradué des études médicales d'une faculté de médecine en Algérie. Pédagogie Médicale 2020. https://doi.org/10.1051/pmed/2020034.

5. Granry JC, Moll M-C. Rapport de mission. État de l'art (national et international) en matière de pratiques de simulation dans le domaine de la santé. Haute Autorité de santé, 2012 [On-line]. Disponible sur: https://www.hassante.fr/upload/docs/application/pdf/2012.

6. Pugh CM. Low-cost, locally fabricated simulators: the wave of the future. J Surg Res 2011;168:29-30. 